\title{
Application of two-dimensional detection based on the background subtraction in badminton Hawkeye system
}

\author{
Li Li ${ }^{1, a}$ \\ ${ }^{1}$ Physical Education Department of Guangxi University, China \\ alili807@foxmail.com
}

Keywords: badminton Hawkeye, background subtraction, two-dimensional detection

\begin{abstract}
With the development of computer technology, computer vision technology was getting more mature. The two-dimensional detection in the badminton Hawkeye system was studied with background subtraction. The mass center coordinates of the badminton were obtained after eliminating the influence of the isolated noise by morphological opening operation, which have found the base of badminton 3D information obtaining and reconstruction for the following research.
\end{abstract}

\section{Introduction}

The two-dimensional detection is one of the most important steps of the badminton Hawkeye system. The mass center coordinates of the badminton obtained by detection are the premise and foundation for three-dimension reconstruction and judging the placement of the badminton.

The background subtraction is used in this paper to detect the two-dimensional information of the badminton Hawkeye system. The background subtraction has been developed advanced in the recent years. For example, Haritaoglu ${ }^{[1]}$ thought that the pixel of the image could be analysis and counted by the maximum time subtraction, the maximum and minimum strength. The analysis model of the data was established and the background was updated. The self-adaptive background model was built with Kalman filtering to test the change of the light by Karmann ${ }^{[2]}$. The change of the light and the interference of the moving background to the image was analyzed with self-adaption Gauss background model by Grimson \& Stauffer ${ }^{[3-4]}$.

\section{Process of two-dimensional detection}

The process of the background subtraction of the badminton Hawkeye system can be divided into the following steps. Firstly, turn on the cameras to take the first frame as the background image. The binary image is obtained after differencing the next images and the background image. Secondly, the number of the characteristic points are statistics, and a counter is set to statistic the number of the changing characteristic points. Thirdly, two threshold are set. One is for division, the counter plus 1 when the absolute value of the difference of the pixel. Another one is for compare with the counter. When the value of the counter is larger than this threshold, it can be confirmed that there's moving object in the image. Last, the differenced images are saved and the mass center are calculated.

\section{Noise processing}

The background subtraction is easily influenced by noise and mutation factor because it only concern about the single pixel, which will distort the detected results. The light in the match is the key factor of detecting and locating. The mathematical morphology is used to eliminate the isolated 
noise of the images.

The basic way of the arithmetic of mathematical morphology is divided into expansion operation and corrosionoperation. Corrosion operation can be expressed by:

$$
E=X \odot B=\left\{(\mathrm{x}, \mathrm{y}) \mid \hat{B_{x, y}} \subseteq X\right\}
$$

Where $\mathrm{X}$ is defined as the image value after binaryzation; $\mathrm{B}$ is defined as the element after corrosion; the output is E, which is also a binaryzation value.

Expansion operation is just contrary to corrosion operation. The empty pixel are filled after image segmentation, which makes the image more full. The expression can be showed as:

$$
D=X \oplus B=\left\{(\mathrm{x}, \mathrm{y}) \mid\left[\hat{B}_{x, y} \cap X \subseteq X\right]\right\}
$$

Where $\mathrm{X}$ is defined as the image value after binaryzation; $\mathrm{B}$ is defined as the element after corrosion; the output is D after expansion operation.

Morphological opening operation refers to corrode the image to eliminate the isolated noise and then expand it to separate the object from the boundary, which makes the boundary smooth and it will fill the empty pixel after corrosion operation. The morphological opening operation could be expressed as :

$$
X \circ B=(\mathrm{X} \odot B) \oplus B
$$

(3) Where $\mathrm{X}$ is

defined as the image value after binaryzation; $\mathrm{B}$ is defined as the element after morphological opening operation.

\section{Results and analysis}

The background subtraction is used to detect the moving badminton in the badminton Hawkeye system. And then the morphological opening operation is used to smooth the images. The fig. 1 shows the results of the experiment.

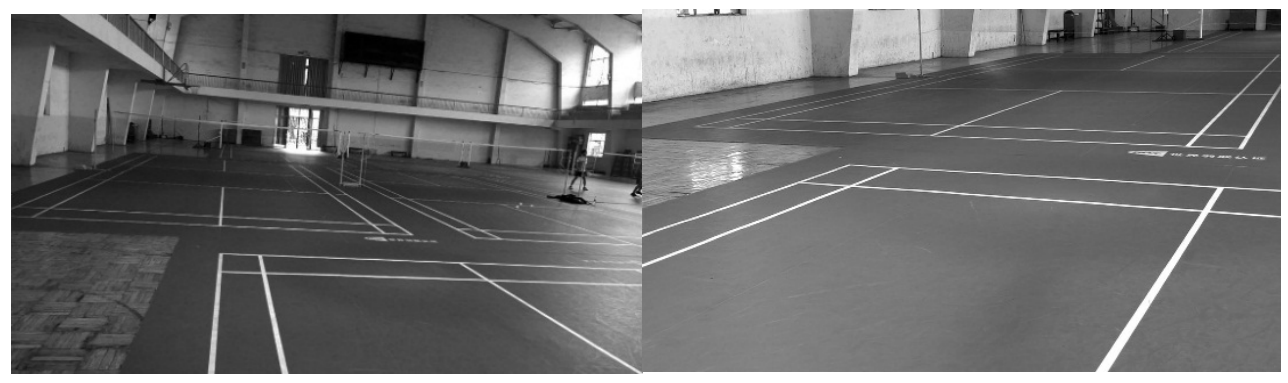

(a1)

(b1)

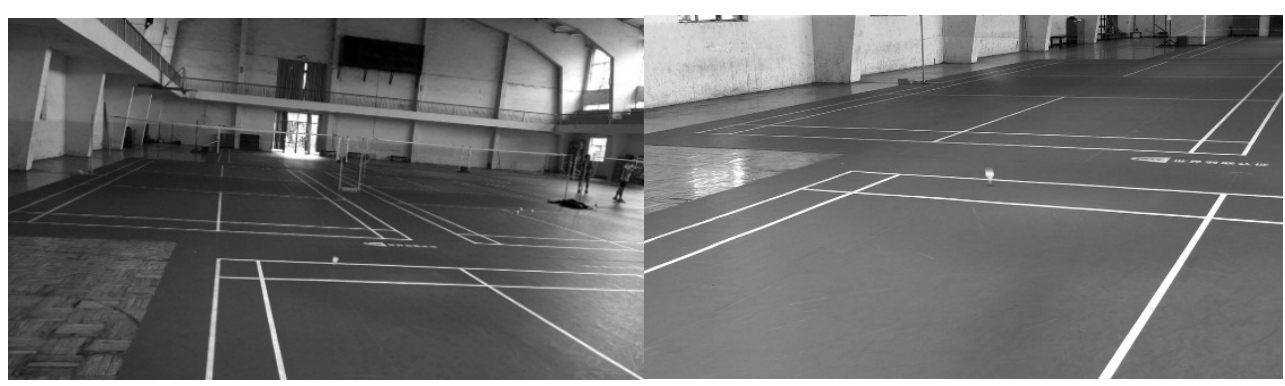

(a2) 


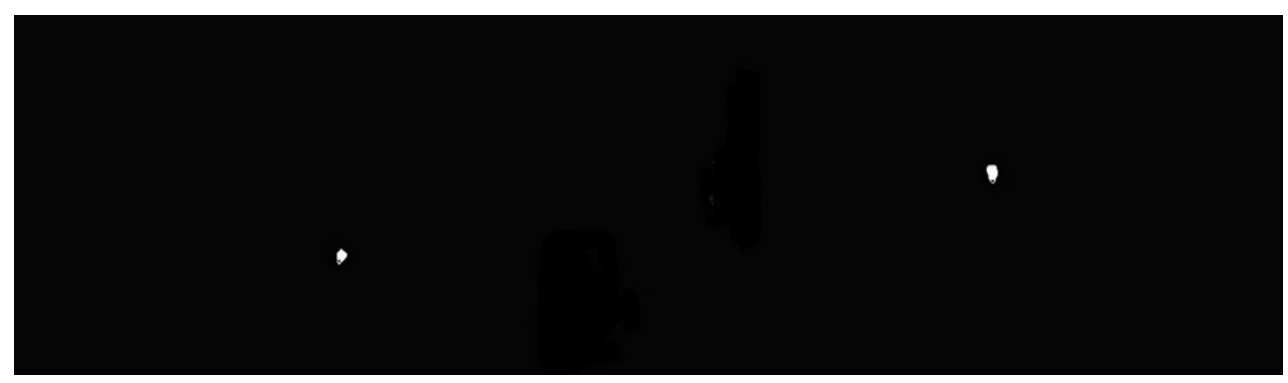

(a3)

(b3)

Fig.1 The results of Location

Where (a1)(b1) are the background images of the left and right camera in the badminton Hawkeye system; (a2) (b2) are the images filmed the moving object(badminton); (a3) (b3) are the images after segmentation. The cross center marked in the image is the mass center of the badminton. The left and right camera could film 8-10 sets images and fig.2 is just one set of them. The mass center of the badminton is showed in table 1.

Table1 Mass center of the badminton

\begin{tabular}{c|c|c|c|c}
\hline \multirow{2}{*}{ NO. } & \multicolumn{2}{|c|}{ Left image } & \multicolumn{2}{c}{ Right image } \\
\cline { 2 - 5 } & X coordinate & Y coordinate & X coordinate & Y coordinate \\
\hline 1 & 720 & 621 & 1200 & 800 \\
\hline 2 & 691 & 562 & 541 & 606 \\
\hline 3 & 665 & 502 & 553 & 576 \\
\hline 4 & 655 & 471 & 567 & 544 \\
\hline 5 & 594 & 384 & 581 & 530 \\
\hline 6 & 574 & 352 & 592 & 499 \\
\hline 7 & 556 & 324 & 611 & 467 \\
\hline 8 & 537 & 298 & 624 & 434 \\
\hline 9 & 504 & 254 & 633 & 401 \\
\hline 10 & 474 & 221 & 649 & 369 \\
\hline 11 & 448 & 194 & 665 & 336 \\
\hline 12 & 441 & 168 & 681 & 310 \\
\hline 13 & 445 & 152 & 695 & 285 \\
\hline 14 & 453 & 131 & 708 & 260 \\
\hline 15 & 465 & 94 & 724 & 233 \\
\hline
\end{tabular}

The first set data in table 1 includes the point(1200, 800). But the size of the image is $1080 \times$ 650 , which we consider that there's no object in the image. In the procession of calculation, the next set data will be read to calculate the three-dimensional information.

According to the previous discussion, the background subtraction and supplemented by morphological opening operation is used to detect the two-dimensional information. The mass center of the badminton in fight is obtained, which have found the base of badminton three-dimensional information obtaining and reconstruction for the following research. 


\section{References}

[1]. Karmann $\mathrm{K}$ and Brandt A. Moving object recognition using an adaptive background memory. In: V Rappellini, Time-varying Image Processing and Moving Object Recognition. 2. Elsevier, Amsterdam, The Netherlands, 1990.

[2]. McKenna Setal, tracking groups of people. Computer Vision and Image Understanding, 2000,80(1):42-56

[3]. Kilger M. A shadow handler in video-based real-time traffic monitoring system. In: Proc IEEE Workshop in Applications of Computer Vision, Palm Springs, CA, 1993:1060-1066

[4]. Haritaoglu I, Harwood D and Davis L. W4: real-time surveillance of people and their activities. IEEE Trans Pattern Analysis and Machine Intelligence,2000,22(8):809-830

[5]. Stauffer C and Grimson W. Adaptive background mixture models for real-time tracking. In: Proc IEEE Conference on Computer Vision and Pattern Recognition, Fort Collins, Colorado, 1999,2:246-256

[6]. Lipton A, Fujiyoshi H and Patil R. Moving target classification and tracking from real-time video. In: Proc IEEE Conference on Computer Vision, Princeton, NJ, 1998,8-14

[7]. Collins R et al. A system for video surveillance and monitoring: Vsam final report, Carnegie Mellon University, Technical Report: CMU-RI-00-12,2000

[8]. FU Si-hua, ZHANG Xiao-hu. Moving targets real-time detecting methods based on the image sequences [J]. Optical Technique, 2004,03 (02):215-217

[9]. CAI Hai-peng. Algorithm and Application of Moving Targets Detection in Video Streams [D]. Hangzhou: Zhejiang University, 2008. 